\title{
Flexible-Wing-Based Micro Air Vehicles
}

\author{
Peter G. Ifju*, David A. Jenkins", Scott Ettingers, Yongsheng Lian ${ }^{\S}$ and Wei Shyy ${ }^{\dagger}$ \\ Department of Aerospace Engineering, Mechanics and Engineering Science \\ University of Florida, Gainesville, FL 32611-6250 \\ Martin R. Waszak \\ Dynamics and Control Branch, NASA Langley Research Center, Hampton, VA 23681
}

\begin{abstract}
This paper documents the development and evaluation of an original flexible-wing-based Micro Air Vehicle (MAV) technology that reduces adverse effects of gusty wind conditions and unsteady aerodynamics, exhibits desirable flight stability, and enhances structural durability. The flexible wing concept has been demonstrated on aircraft with wingspans ranging from 18 inches to 5 inches. Salient features of the flexible-wing-based MAV, including the vehicle concept, flexible wing design, novel fabrication methods, aerodynamic assessment, and flight data analysis are presented.
\end{abstract}

\section{Background}

Micro air vehicles, or "MAVs", belong to a class of aircraft currently designated with a maximum size of about 6 inches and are capable of operating at speeds of $25 \mathrm{mph}$ or less. The concept is for a small, inexpensive and expendable platform that can be used for missions of surveillance and measurements in situations where larger vehicles are not practical. Practical applications of MAVs are becoming more achievable with the ever-decreasing size and weight of the payload components that can include video cameras, chemical sensors, electronics, and communication devices. Only a few years ago the thought of a 6-inch flying machine equipped with a functional video camera was science fiction. Today it is a demonstrated fact. A number of aspects related to MAVs have been investigated [see, e.g., 1-9].

It is well known that flight in the Reynolds number range between 10,000 and 100,000 , flow separation around an airfoil can lead to sudden increases in drag and loss of efficiency. In nature, one can observe the relationship

\footnotetext{
*Associate Professor.

§Graduate Student.

${ }^{\dagger}$ Professor and Chairman, Fellow AlAA.

${ }^{t}$ Senior Research Engineer, Senior Member AlAA
}

Copyright 2002 by Authors, Published by the American Institute of Aeronautics and Astronautics, Inc with permission. between Reynolds number and aerodynamic efficiency in birds where large species soar for extended periods of time while small birds have to flap vigorously (high frequency) to remain airborne. The Reynolds numbers of the larger species are well above 100,000 whereas hummingbirds would fly at below 10,000 if they attempted to soar. Additionally, the wing loading for small birds is less than that for large birds.

In the quest to develop practical MAVs, two approaches have been followed so far. The first and most popular is to configure the airframe as a lifting body or flying wing using conventional propeller driven thrust. In this approach, the emphasis is to increase the relative area of the lifting surface while decreasing drag, directly addressing the decrease in the aerodynamic efficiency, and ignoring issues of stability and control. In nature, the great optimizer, there are no examples of lifting bodies or flying wings. All birds and bats have well defined wings and a fuselage that blend together. The second approach that has been explored on the MAV scale is the direct mimicry of birds $[6,10]$. By flapping, birds produce both lift and thrust. Researchers have demonstrated flapping mechanisms in the lab environment, but have yet to produce efficient flying machines. Complex control issues and high power consumption remain as formidable challenges for this type of MAV.

Conventional approaches have used optimized rigid wings and accepted the need for enhanced stabilization systems or supreme pilot skill to deal with the intrinsically unsteady behavior. Noticeable successful examples of MAVs include Aerovironment's "Black Widow", an electric 6inch rigid-wing design [1], and the flexible-wing design by the University of Florida group. Other successful examples of rigid wing designs include the "Trochoid" [7] developed by Steve Morris of MLB Company and Sander's "Microstar", another DARPA-funded MAV. Both of these also have gyro assisted stabilization systems. Without these enhancements, lifting bodies are difficult to control. This difficulty was vividly demonstrated at the recent International Micro Air Vehicle competition held in 
Gainesville, FL. Of the nine official entries, none of the conventional rigid-winged aircraft able to complete the mission was smaller than 9 inches (maximum dimension).

Previous studies, documented in Waszak et al. [9], Shyy et al. [8, 11, 12], Smith and Shyy [13], and Jenkins et al. [14], indicate that an alternate approach, specifically letting the lifting surface move and deform, can lead to more favorable aerodynamic performance in a fluctuating low Reynolds number environment. This aspect is considered critical for MAVs because under the given flight speed, vehicle dimension, and weight, wind speed can change the flight Reynolds number by more than $30 \%$, creating a highly unsteady flight environment. The prior research on membrane-based flexible airfoil has helped lead to our flexible wing MAV concept, which we have been applying and improving over the past three years. We utilize conventional propeller driven thrust in combination with an adaptive-shape, flexible wing that adapts to flight conditions and also develops a stable limit cycle oscillation during flight. We believe that the behavior of our flexible wing is an enabling technology that will lead to practical micro air vehicles in the future.

The present flexible wing technologies are developed to produce smooth flight even in gusty wind conditions. It is our view that in order to produce the best overall flight characteristics, one must first start with an airplane that is intrinsically stable. This goal is accomplished via the adaptive nature of the wing as well as its natural oscillation. Our aircraft can be flown by novice to average RC pilots, without the aid of gyro enhanced stabilization. We have demonstrated the merits of these MAVs at the International Micro Air Vehicle Competition by winning the event the last three years in a row. We have successfully demonstrated MAVs with a maximum dimension as small as 5 -inches.

\section{The Vehicle Concept}

The development of our flexible wing utilizes a combination of biologically inspired design and the incorporation of modern composite materials. The wing is thin and under-cambered as are those of small birds and bats. The micro air vehicle that we have developed is constructed with a carbon fiber skeleton and thin membrane materials. In the fuselage we use carbon fiber prepreg cloth and on the wing we use an extensible latex rubber membrane. The configuration is similar to a blended wing-body where the fuselage blends into the wing similar to that of birds and bats. The MAV shown in Figure 1 is the product of more than one year of design iteration using flight tests and pilot feedback as the primary method of evaluation.
Figure 2 shows video footage taken from the ground of our MAV. The insert shows the view from the on-board video camera.

The shape of the wing allows for the maximum lifting surface while staying within a 6 -inch diameter sphere. In order to define the design space for our flexible wing we built numerous prototypes to learn how the geometry of the carbon fiber skeleton affects the flight characteristics.

We also varied the relative stiffness of the different parts of the skeleton. In Figure 3 we show 24 of the designs that were successfully flight-tested. We were able to make observations in the field in order to qualitatively rank their performance. Using this relatively crude trial and error process, we were able to identify the configurations that provided the best performance.

In order to explore the limit of current technologies and to facilitate vehicle development in a timely fashion, our strategy to date has been to use off-the-shelf components. A typical 6-inch MAV, with an electric motor driven by lithium polymer prismatic batteries, at airspeeds between 15 and $25 \mathrm{mph}$ can fly for up to fifteen minutes. With the latest battery technology, much longer flight time can be attained. The overall flying weight is $\mathbf{5 2}$ grams with a camera.

\section{Flexible Wing Design}

The flexible nature of the wings can provide several nonobvious advantages over their conventional rigid counterparts. The wings that we have fabricated with a carbon fiber skeleton and extensible latex rubber skin have the ability to adapt to the airflow to provide smoother flight. This is accomplished via the passive mechanism of adaptive washout. In sailing vessels adaptive washout is produced through twist of the sail. This greatly extends the wind range of the sail and produces more constant thrust (lift), even in gusty wind conditions. In the wings that we have designed, the shape changes as a function of the airspeed and the angle of attack. The adaptive washout is produced through extension of the membrane and twisting of the framework, resulting in angle of attack changes as well as decambering along the length of the wing in response to air speed and overall angle of attack. For example, as the plane hits a head-on wind gust the airspeed suddenly increases. The increased airspeed causes a shape change in the wing that decreases the lifting efficiency, but because the airspeed in the gust is higher, the wing maintains nearly the same lift. Once the airspeed decreases, the wing recovers to the original configuration. If there is a decrease in the relative airspeed, the angle of attack increases and the wing becomes more efficient and near 
constant lift is restored. The net result is a wing that flies with exceptional smoothness, even in gusty wind conditions. The adaptive washout mechanism need be tuned into the wings in order to work effectively. We have built hundreds of wing configurations and have been able to produce many wings with remarkably smooth flying characteristics. Figure 4 illustrates the flexible nature of our wing.

For aircraft with very small inertia, as in the case of MAVs, changes in wing loading can immediately affect the flight path. The need for suppressing the effects of wind gusts becomes more critical when the aircraft becomes smaller and lighter, especially if it is to be used as a camera platform. Additionally, as the airspeed of the vehicle decreases, wind gusts become a larger percentage of the mean airspeed of the vehicle. For example, our 6-inch aircraft flies between 15 and $25 \mathrm{mph}$. On a typical day the wind speed can vary by more than $10 \mathrm{mph}$. For rigid wings, the lift can vary by $50 \%$ or more over the short period of time during the gust. To make matters more critical, gusts are not always head-on. Because control of these aircraft is one of the most important hurdles, it is critical to suppress unwanted and sudden changes in direction, elevation and orientation.

\section{MAV Fabrication Methods}

In the early stages of the development of our MAV designs we relied heavily on an Edisonian approach. Our philosophy was simply to build many designs and flighttest them while carefully observing their flight characteristics. In order to use this approach we made some significant advances in the construction methods so that design iterations could be made quickly and each design could be thoroughly tested. The construction methods developed for this project were the enabling technology that allowed us to implement our designs. We make our airframes using unidirectional carbon fiber prepreg, woven carbon fiber prepreg, Kevlar thread, and tough mono-film materials. Most of the materials are integrated and vacuum bag cured all at once. Each aircraft can be designed, built and ready to fly within five man-hours. The resulting MAVs are nearly indestructible (since they have no landing gear this is a must), yet are as light as the conventional balsa wood counterpart. Each design is flight-tested and evaluated by the pilots and observers for flight characteristics including stability of flight, payload capacity and maneuverability.

Step-by-step construction techniques used to fabricate a MAV wing are described here.

\section{Wing Construction}

Step 1. A drawing is made of the wing planform to act as a guide for carbon fiber placement.

Step 2. The drawing is taped onto a curved tool.

Step 3. A layer of nonporous Teflon release film is placed over the drawing.

Step 4. Unidirectional carbon fiber tape is cut into long narrow tacky strips.

Step 5. The carbon fiber strips are placed on the release film using the drawing as a guide. Multiple layers are used in places where high stiffness is required. Overlap at the corners assures a mechanically sound joint.

Step 6. Nonporous Teflon release film is then placed over the assembly.

Step 7. The assembly is then placed into a vacuum bag and subsequently into a vacuum oven for cure.

Step 8. After the cure cycle is complete, the carbon fiber wing skeleton in separated from the tool.

Step 9. Spray mount adhesive is applied to the skeleton.

Step 10. Thin latex nubber material is then applied to the wing.

Step 11. Cyanoacrylate adhesive is used to reinforce the bond line.

Step 12. Excess latex rubber is trimmed away.

\section{Aerodynamic Assessment}

For a rigid wing, the pressure distribution is determined by the wing shape and free-stream flow properties. For a flexible wing, its shape changes under aerodynamic load, and, consequently, the angle of attack and surface pressure distribution will change along with the flight environment. In order to shed light on the aerodynamic characteristics of membrane wing, one needs to solve coupled fluid-solid dynamics to track both the shape change and the pressure distribution on the wing shape.

Even though the importance of the viscous effect on membrane wing aerodynamics has been recognized for quite some time (Nielsen [16]), few works have been published which address the issue. To date, most of the works in membrane wing aerodynamics is based on simplified fluid and structure models [15]. The first use of Navier-Stokes equations as the flow dynamics model in a membrane wing theory appears to be the work of Smith and Shyy [17]. In their work a computational procedure is presented that models the interaction of a two-dimensional flexible membrane wing and laminar, high-Reynoldsnumber steady fluid flow. Results from the viscous flowbased membrane wing model were compared with a potential flow based membrane wing theory. Unsteady laminar flow surrounding membrane wing has been reported by Shyy and Smith [18], and a corresponding turbulent flow computation by Smith and Shyy [19]. Recently, Jackson reported an analysis to address the 
aerodynamics of high-aspect ratio membrane wings of conical shape using the lifting-line and thin-airfoil theories. Aerodynamics and optimization of low Reynolds number flexible wing are reported by $[8,20,21,22]$. In the following, we use the CFD simulations to highlight the aerodynamics of a representative wing.

The Navier-Stokes equations for incompressible fluids, written in three-dimensional curvilinear coordinates [23], are solved using a multi-grid-block, pressure-based, moving grid technique $[12,22]$. To facilitate the solution of such moving boundary problems, we have implemented an automated regridding procedure to ensure that the grid system not only matches the geometric changes but also is smooth and not excessively skewed.

Obviously, our goal in not only to compute and analyze the dynamics of the coupled fluid and structure systems, but also to use the knowledge gained to improve our design capability. Accordingly, shape optimization has also been conducted based on the CFD solutions. To facilitate such an optimization task, we adopt a gradient-based search technique [24]. From the initial condition and the gradient information obtained in the course of computation, the shape will be progressively modified toward the estimated optimal target. Such procedures require the generation of a series of new grid systems based on the new geometries. The present moving grid technique can perform that task effectively because the remeshing process can be handled with exactly the same procedure as the moving boundary problem, and with the same automation. The 3-D flexible wing aerodynamics and shape optimization efforts are ongoing.

To illustrate the aerodynamic characteristics associated with MAVs, a schematic of the wing geometry, along with representative grid layout for $C F D$ simulations, and the pressure contours on upper and lower wing surfaces are shown in Figure 6. For the present case, the chord Reynolds number is $6 \times 10^{4}$ and the angle-of-attack is $6^{\circ}$. It is interesting to see that while the pressure field is clearly three-dimensional, the distribution largely follows the geometric definition of the wing. It is well known $[25,26]$ that the rates of change of the lift and drag coefficients with angle-of-attack are strongly affected by the aspect ratio of the wing. Specifically, existing evidence, all based on high Reynolds number testing, indicates that the wings of various aspect ratios have about the same angle-of-attack at zero lift, but the slope of the lift curve increases progressively with increase of aspect ratio.

Streamlines at an angle of attack of $6^{\circ}$ are shown in Figure 7. Detailed flow structures including trailing vortex lines are clearly visible. The aerodynamic assessment has demonstrated that at the designated Reynolds number range, the lift is sufficient to support the current design. With the flexible wing technology, the lift can be maintained with reduced influence from the unsteady flight environment.

\section{Wind Tunnel Test}

A wind tunnel test was performed to provide data with which to investigate the benefits of the aeroelastic wing concept. The wind tunnel test was conducted in the Basic Aerodynamics Research Tunnel (BART) at NASA Langley Research Center. A variety of data were collected to aid in the study of the vehicle's dynamics and control properties and consisted of aerodynamic force and moment data, static and dynamic wing deformation data, and flow visualization using smoke. These data were collected for a rigid wing and three different batten/membrane arrangements over a range of operating conditions determined by dynamic pressure, power setting, vehicle attitude, and control surface deflection.

The different batten arrangements are depicted in Figure 8 . The one-batten design has the most flexibility and larger membrane stretch. The two-batten design is, by comparison, stiffer and exhibits less membrane stretch under aerodynamic load. Both wings were tested using a 4 mil latex membrane. The six-batten wing was covered with an inextensible monofilm membrane that further increased the stiffness of the wing and exhibited less membrane deformation and vibration. The rigid wing was constructed of a two-batten frame covered with a graphite sheet.

The results indicate that the elastic membrane wing allows the vehicle to achieve higher angles of attack without stalling (see Figure 9). This fact coincides with significant static deformation of the wing under load, particularly at higher angles of attack (AoA), and is accompanied by extensive high frequency membrane vibration. The static deformation allows the wing to see a smaller effective angle of attack at high vehicle attitudes (see Figure 10). Flow visualization suggests that the wing deformation contributes to weaker wing tip vortices. It is likely that there is some link between the vortex strength and structure, membrane billowing, and the stall resistance of the elastic membrane wings.

The vehicle was shown to be statically stable in all axes and that the non-dimensional static stability derivatives of the vehicle were found to be generally larger than for typical piloted aircraft. Because the vehicle has been "tuned" using flight test experiments it is likely that the relatively large pitch and yaw stability and large dihedral 
effect enhance the vehicle's flying qualities. The vehicle trims near the maximum $L / D$, but the maximum $L / D$ is significantly lower than other comparable micro aerial vehicles.

Additional investigation of the stability and control properties of the vehicle is underway. These studies will emphasize the development of additional understanding of the physical properties of the membrane wing concepts and use this understanding to improve the design of the vehicle.

\section{Analysis of Flight Test Data}

Pilot reports and video recording through a small on-board camera indicate that our flexible-wing micro air vehicles have unusually smooth flying characteristics, both during visual contact flying in the conventional RC mode at close range and also when flying more remotely using the transmitted video from the on-board camera. We consider the smooth flying qualities to be an important characteristic of practical micro air vehicles in the future, even when they will be made to fly autonomously. In the continuing design process we are striving to make the feedback information concerning handling qualities more objective and quantitative, rather than relying solely on the pilot's informal comments. Although using verbal feedback has been successful so far, our ability to evaluate the effects of design changes is limited and often inconclusive. To address this shortcoming, we have developed a system for recording the pilot's control inputs during the entire flight and we are developing tools for objective interpretation of this data.

Most of our test flying is done using conventional RC equipment at close range, keeping the vehicle in continuous visual contact. Because of the small size of the vehicles, flying at distances greater than about 100 feet can quickly cause loss of orientation unless the pilot is flying by monitoring the video output from an on-board camera. The $\mathrm{RC}$ transmitter produces a radio frequency signal that causes the RC receiver carried in the vehicle to develop a series of pulses of varying pulsewidths (pulse width modulated or PWM) that are delivered to the control surface servos as the command signals for the desired positions of these surfaces. On the equipment we use, the pulses are generated at a constant frequency of $40 \mathrm{~Hz}$. To capture this control input information, we have developed a simple system that uses a second $\mathrm{RC}$ receiver on the same $\mathrm{RC}$ frequency as the flight unit to monitor the pulse widths of the servo signals on the various servo terminals on the receiver and store the data on a notebook PC (see Figure 11). With this system, stick input data is recorded without any contact or interference with the pilot or the micro air vehicle and the recording can be done at any reasonable range within the operating range of the RC system (at least

\section{0 meters)}

We found that the most effective representation of the data is in the form of autospectra of the stick deflection rate, as shown in Figure 12. These views allow the pilot's workload directed toward planned maneuvering (in the $0 \sim 1$ $\mathrm{Hz}$ range) and the pilot's workload directed toward stabilization of the vehicle $(1 \sim 10 \mathrm{~Hz}$ range) to be clearly observed and compared. In a preliminary series of test flights, both flexible and rigid wings were evaluated. "Case 1: Flexible wing forward CG" refers to a 10-inch size vehicle with the balance point set to produce reasonably good flying characteristics. "Case 4: Flexible wing forward CG gusty" refers to the same set up but tested on a particularly gusty day. The test identified as "Case 2: Flexible wing aft CG" refers to the $1 / 2$ inch aft CG configuration that leads to pitch instability and requires an obvious increase in the pilot's workload. "Case 3: Rigid wing forward CG" indicates that a rigid wing of the same planform, camber and thickness was substituted for the flexible wing. This wing was made using the same framework of carbon fiber members as the flexible wing, but with a single layer of cured, woven carbon fiber cloth substituted for the flexible membrane. Of particular importance here is the comparison of Case 1 and Case 2 with the rigid wing Case 3 . The rigid wing vehicle in Case 3 displays the same extra and undesirable stabilization workload as the intentionally unstable and hard-to-fly vehicle in Case 2, and thus supports the notion that flexible wings offer measurable stability and ease of control advantages.

\section{Summary and Conclusions}

In the present paper, we highlight the recent research and development in establishing a flexible-wing-based technology for MAVs. Our effort addresses the entire scope comprehensively, including basic concept, novel fabrication approaches, aerodynamics investigations, and flight test data. The outcome is improved understanding of the key issues related to robust and stable flight and vehicle durability. In addition, from the education viewpoint, MAVs offers an excellent opportunity to integrate original research with direct and meaningful student participation. A substantial number of undergraduate and graduate students have been involved in our efforts in the last 5 years. To help foster information exchange and technological advancement, the University of Florida initiated the International Micro Air Vehicle Flight Competition. The event, held annually for the last five years, has attracted participants from a number of countries, and offers a friendly but serious environment to motivate the development of MAVs as integrated endeavors in science and technology. It is our belief that 
the flexible wing offers advantages for stable flight under varying flight conditions. Further progress based on this concept is expected to take place rapidly.

\section{Acknowledgment}

Different aspects of the work reported here have been supported by DARPA, AFOSR, NASA, Lockheed Sanders, Boeing, and ITRI in Taiwan. We also acknowledge useful communication with colleagues in NRL and University of Florida.

\section{References}

[1] Grasmeyer, J.M. and Keennon, M.T., "Development of the Black Widow Micro Air Vehicle," AIAA Paper No. 2001-0127, 2001.

[2] Jones, K.D., Duggan, S.J. and Platzer, M.F., "FlappingWing Propulsion for a Micro Air Vehicle," AIAA Paper No. 2001-0126, 2001.

[3] Ramamurti, R., Sandberg, W. and Lohner, R., "Simulation of the Dynamics of Micro Air Vehicles," AIAA Paper No. 2000-0896, 2000.

[4] Washabaugh, P.D., MacDermid, T., Müller , M.O. Bernal, L.P., Parvi, B.A., Chou, T.K., Zhang, C., and Najafi, K., "An Approach Toward a Wafer Integrated Micro Air Vehicle," Proceedings of Unmanned Air Vehicles Systems, $15^{\text {th }}$ Bristol International Conference, $10^{\text {th }}-12^{\text {th }}$ April 2000, Bristol, UK., 2000.

[5] Guan, W.L., Hsiao, F.B., Ho, C.S., and Huang, J.M., "Development of Low-Cost Differential Global Positioning System for Remotely Piloted Vehicles," AIAA Journal of Aircraft, Vol. 36, No. 4, pp. 617-625, July-August, 1999.

[6] Frampton, K. D., Goldfarb, M., Monopoli, D., and Cveticanin, D., "Passive Aeroelastic Tailoring for Optimal Flapping Wings," Proceeding of the Fixed, Flapping and Rotary Wing Vehicles at Very Low Reynolds Numbers, pp.26-33, 2000.

[7] Morris, S., Holden, M., "Design of Micro Air Vehicles and Flight Test Validation," Proceeding of the Fixed, Flapping and Rotary Wing Vehicles at Very Low Reynolds Numbers, pp.153-176, 2000.

[8] Shyy, W., Berg, M., Ljungqvist, D., "Flapping and Flexible Wings for Biological and Micro Vehicles," Process in Aerospace Sciences, Vol. 35, No. 5, 1999, pp. 455-506.

[9] Waszak, M. R., Jenkins, L. N., and Ifju, P. G., "Stability and Control Properties of an Aeroelastic Fixed Wing Micro Aerial Vehicle," AIAA 2001-4005.
[10] Ellington, C. P., "The Aerodynamics of Hovering Flight," Philosophical Transactions of the Royal Society of London, Vol. 305, No. 1122, pp. 1-181, 1984.

[11] Shyy, W., Thakur, S.S., Ouyang, H., Liu, J. and Blosch, E., Computational Techniques for Complex Transport Phenomena, Cambridge University Press, New York, 1997.

[12] Shyy, W., Udaykumar, H. S., Rao, M.M and Smith, R. W., Computational Fluid Dynamics with Moving Boundaries, Taylor \& Francis, Washington, DC, (1996, revised printing $1997 \&$ 1998) xviii +285 pages.

[13] Smith, R. W., and Shyy, W., "Computational Model of Flexible Membrane Wings in Steady Laminar Flow," ALAA Journal, Vol. 33, No. 10, 1995, pp. 1769-77.

[14] Jenkins D. A., Shyy, W., Sloan, J., Klevebring, F., and Nilsson, M., "Airfoil Performance at Low Reynolds Numbers for Micro Air Vehicle Applications," Thirteenth Bristol International RPV/UAV Conference, University of Bristol, 1998.

[15] Jackson, P. S., and Christie, G. W., "Numerical Analysis of Three-Dimensional Elastic Membrane Wings," AIAA Journal, Vol. 25, No. 5, pp. 676-682, 1987.

[16] Nielsen, J. N., "Theory of Flexible Aerodynamics Surfaces," Journal of Applied Mechanics, Vol. 30, 1963, pp. $435-442$.

[17] Smith, R.W. and Shyy, W., "Computation of Unsteady Laminar Flow Over a Flexible Two-Dimensional Membrane Wing," Physics of Fluids, Vol. 7, 1995, pp. 2175-2184.

[18] Shyy, W., and Smith, R. W., "Computation of Laminar Flow and Flexible Structure Interaction," Computational Fluid Dynamics Review (Ed. Hafez, M., and Oshima, K.), John Wiley \& Sons, 1995.

[19] Smith, R. W., and Shyy, W., "Computation of Aerodynamics Coefficients for a Flexible Membrane Airfoil in Turbulent Flow: A Comparison with Classical Theory," Phys. Fluids, Vol. 8, No. 12, 1996.

[20] Shyy, W., Klevebring, F., Nilsson, M., Sloan, J., Carroll, B. and Fuentes, C. "A Study of Rigid and Flexible Low Reynolds Number Airfoils," Journal of Aircraft, Vol. 36, 1999, pp.523-529.

[21] Levin, O. and Shyy, W., "Optimization of a Flexible Low Reynolds Number Airfoil," AIAA 39th Aerospace Sciences Meeting \& Exhibit, Paper No. 2001-0125, 2001. 
[22] Lian, Y., Steen, J., Trygg-Wilander, M. and Shyy, W., "Low Reynolds Number Turbulent Flows Around a Dynamically-Shaped Airfoil," AIAA $31^{\text {st }}$ Fluid Dynamics Conference, Paper No. 2001-2723, 2001.

[23] Shyy, W., Computational Modeling for Fluid Flow and Interfacial Transport, Elsevier, Amsterdam, The Netherlands, (1994, revised printing 1997) xviii +504 pages.

[24] Design Optimization Tools, Vanderplaats Research \& Development, Inc., 1999.
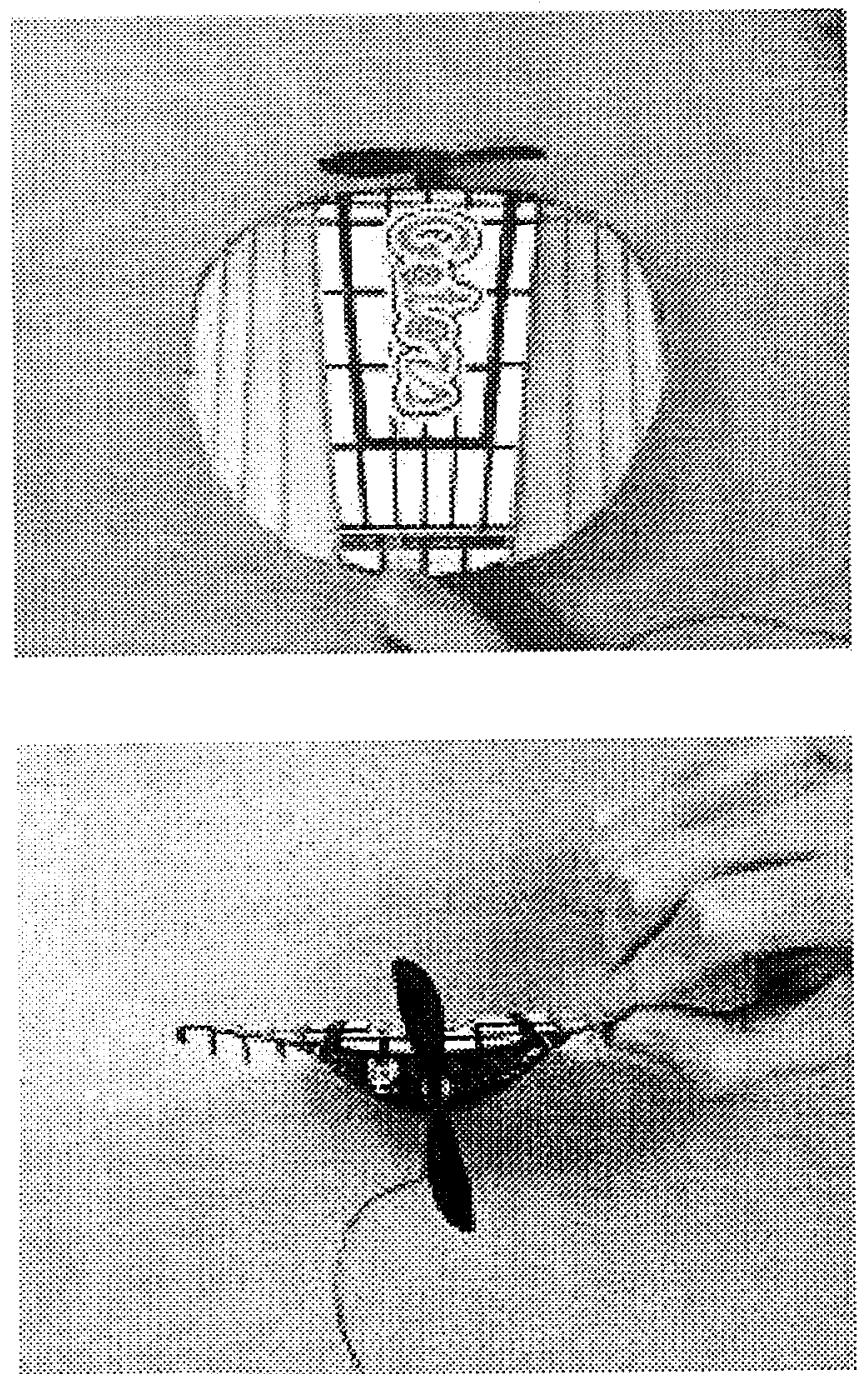

[25] Prandtl, L, and Tietjens, O. G., Applied hydro- and aero- mechanics, 1934, Reissued by Dover, Now York, 1957

[26] Abbott, I. H., and Von Doenhoff, A. E., Theory of Wing Sections, New York, Dover, 1959.

[27] Shyy, W., Jenkins, D. A. and Smith, R. W., "Study of Adaptive Shape Airfoils at Low Reynolds Number in Oscillatory Flows," AIAA Journal, Vol. 35, 1997, pp.15451548.
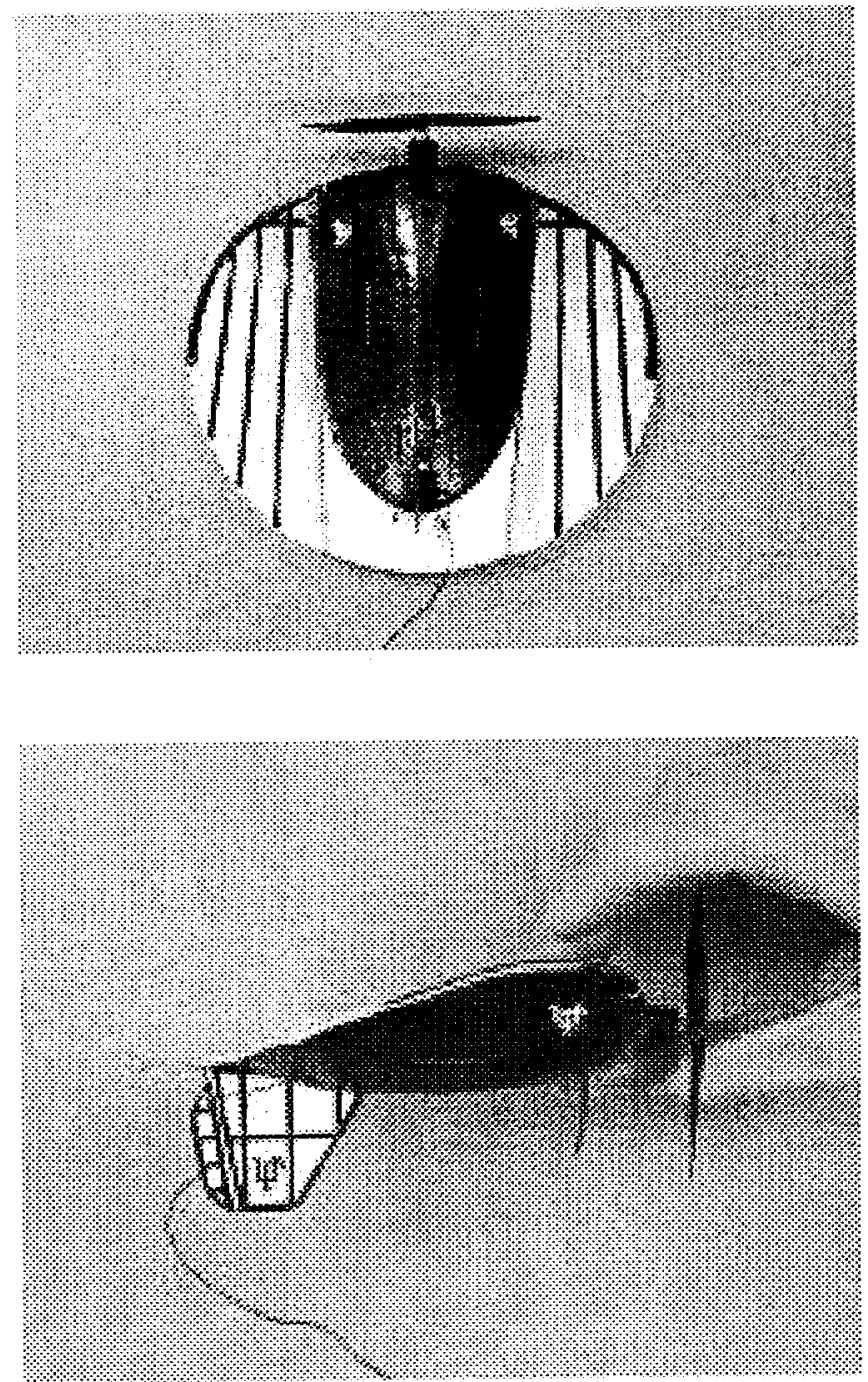

Figure 1. The 6-inch maximum dimension MAV with video camera. 


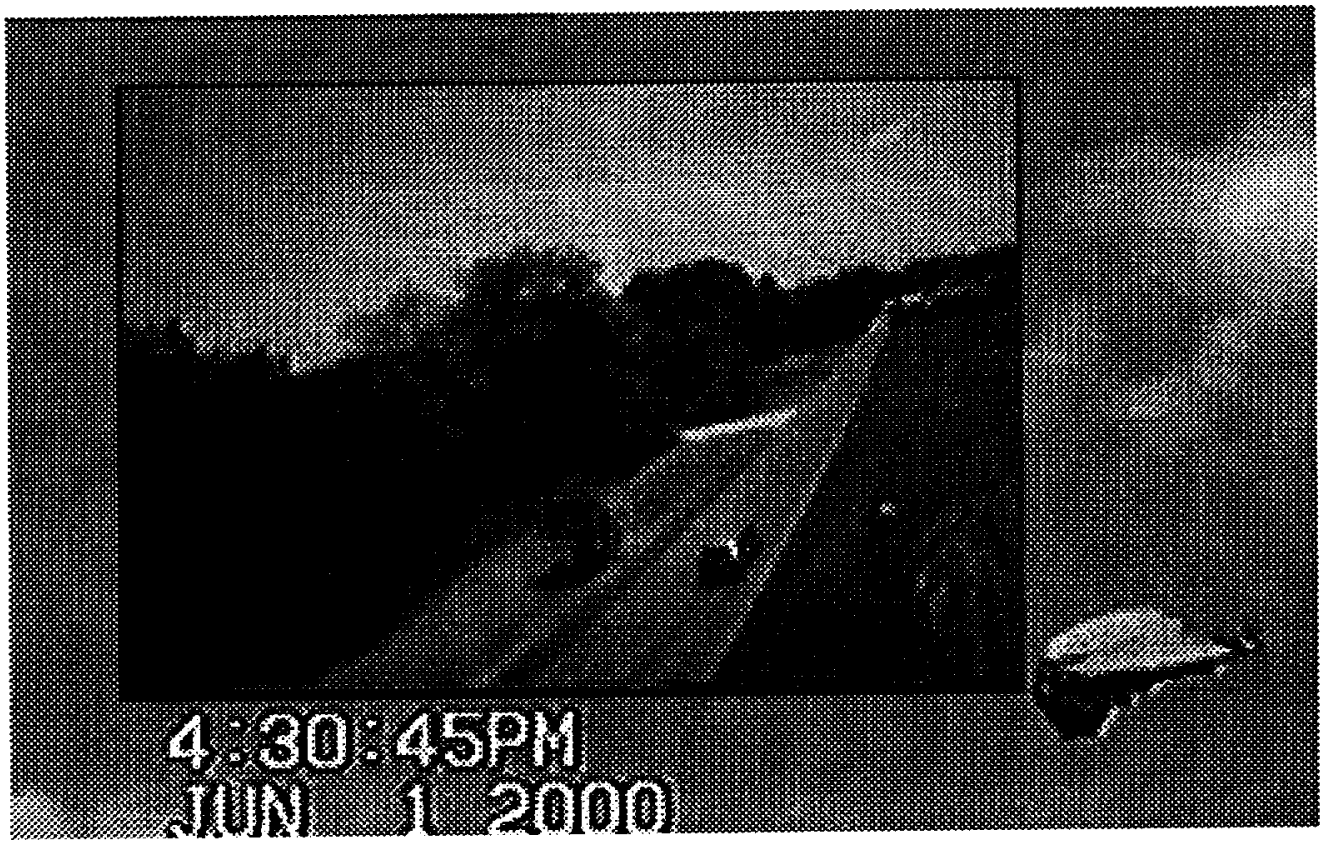

Figure 2.View of MAV from the ground and video footage from on board.

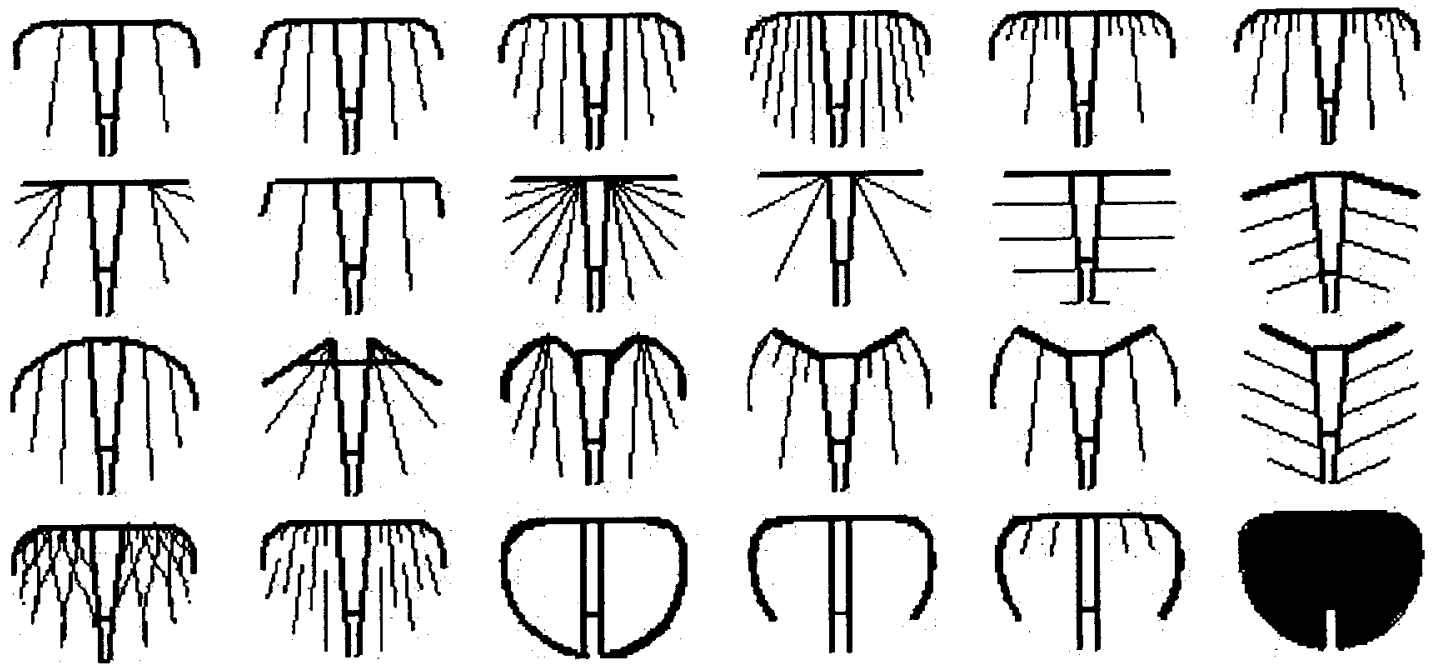

Figure 3. Illustration of different carbon fiber skeletons tested in flexible wing development. 


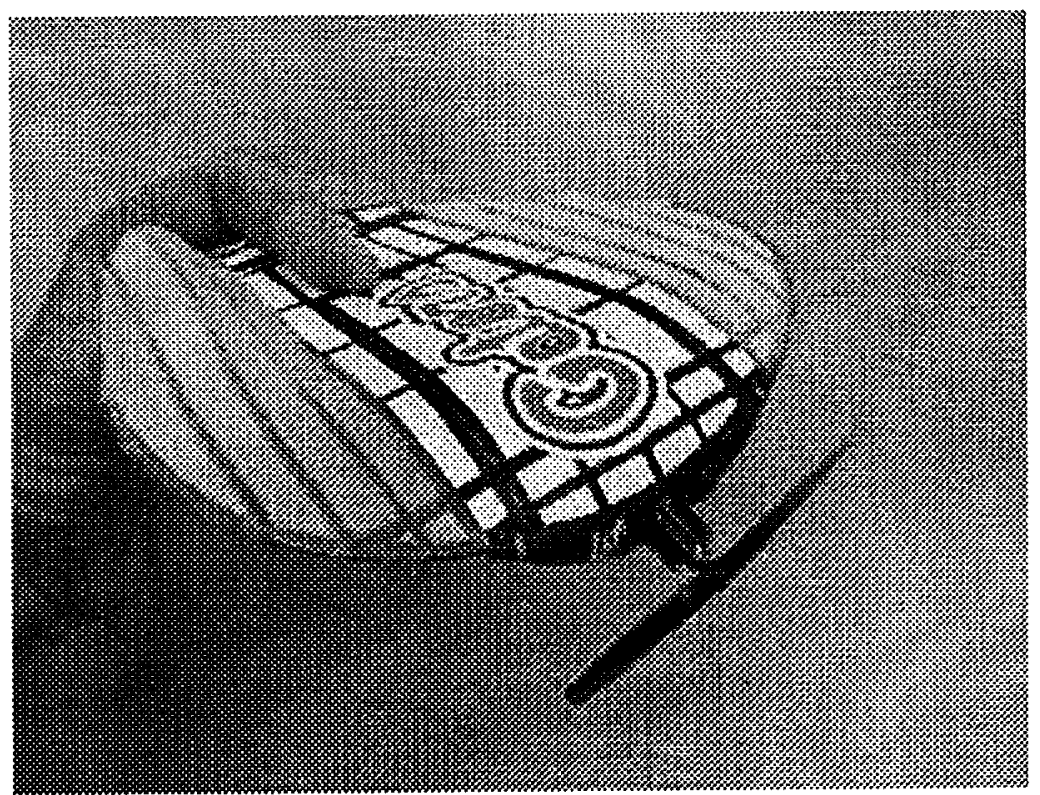

Figure 4. The wings flex, even under small aerodynamic loads.
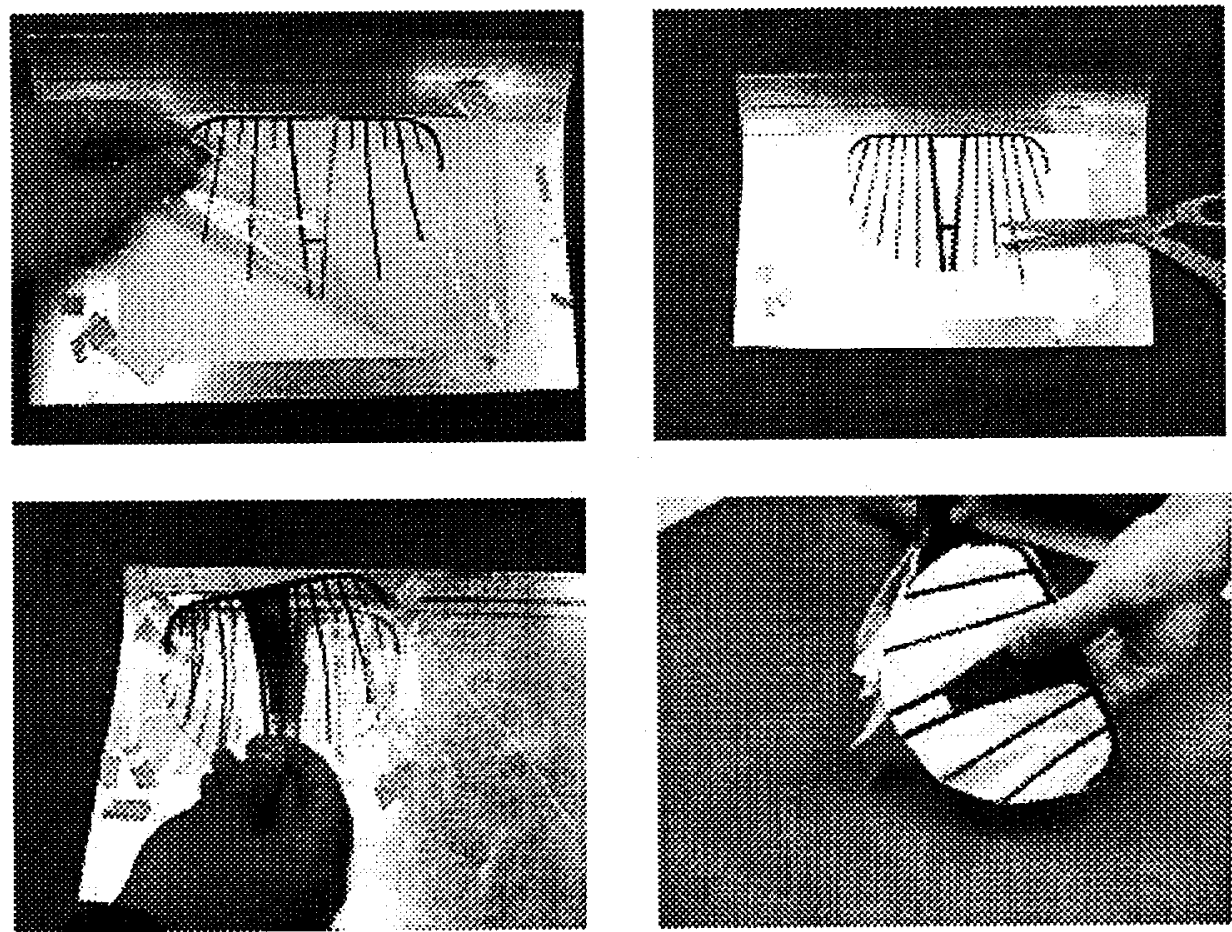

Figure 5. Illustration of the wing construction process. 


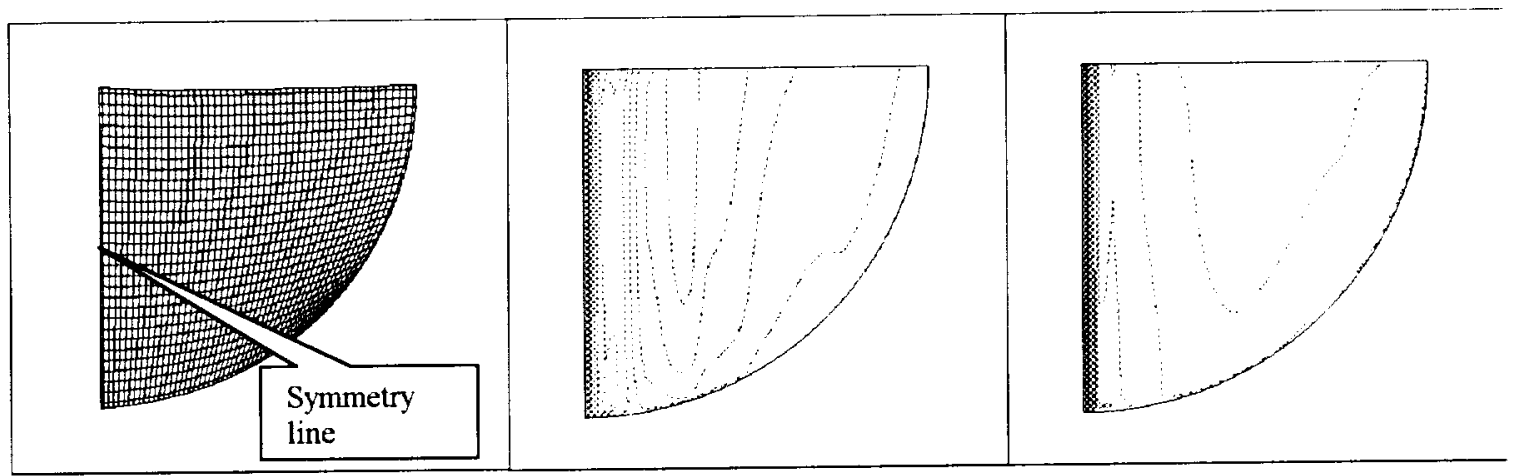

Figure 6. The schematic of the wing geometry, shown with representative grid layout for CFD simulations, and the pressure contours on upper and lower wing surfaces at angle of attack of $6^{\circ}$. The camber Reynolds number is $6 \times 10^{4}$.

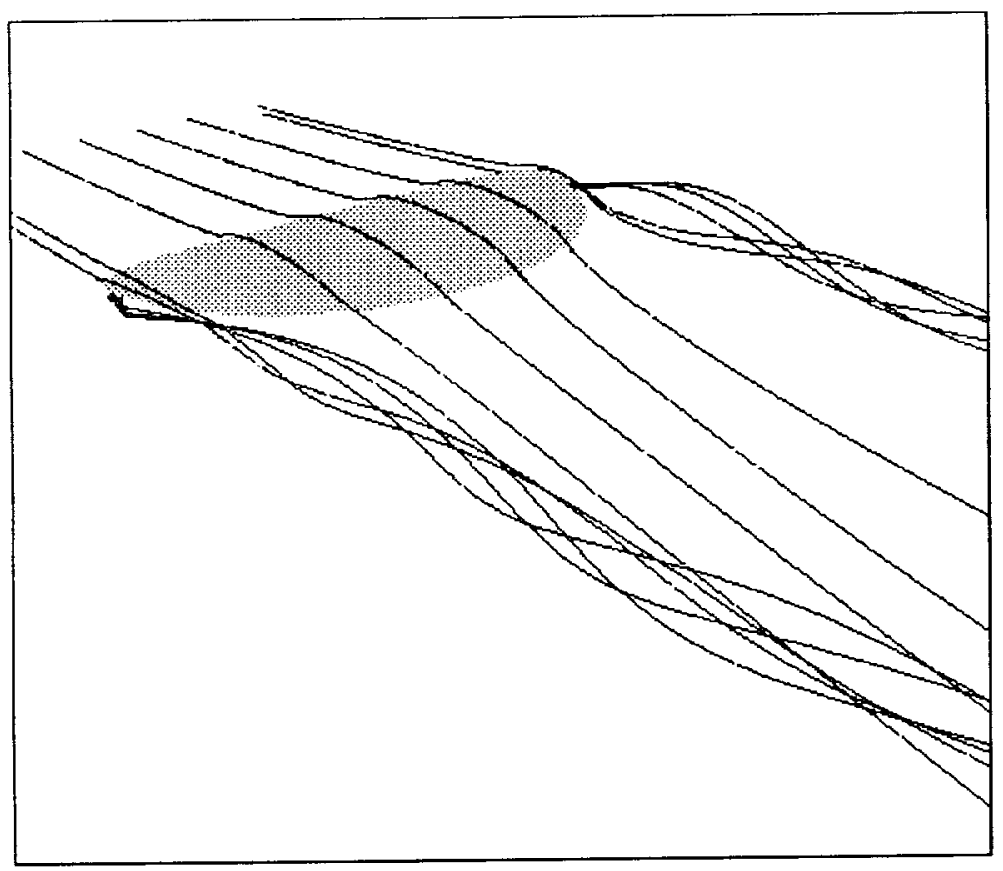

Figure 7. Streamlines of a representative wing at angle of attack $6^{\circ}$. 


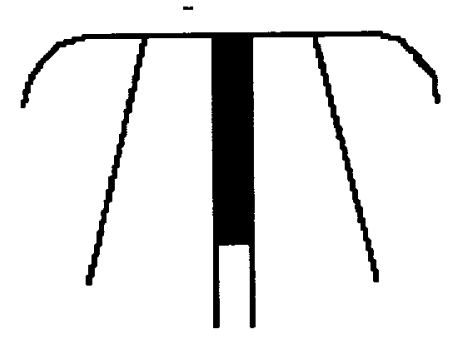

(i)

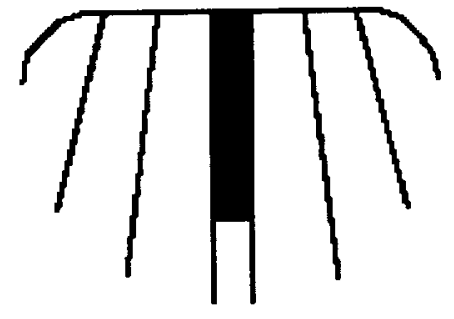

(b)

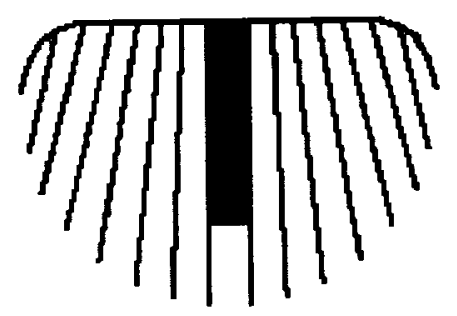

(c)

Figure 8. Batten arrangement for membrane wings.

(a) One-batten, (b) two-batten, (c) six-batten.

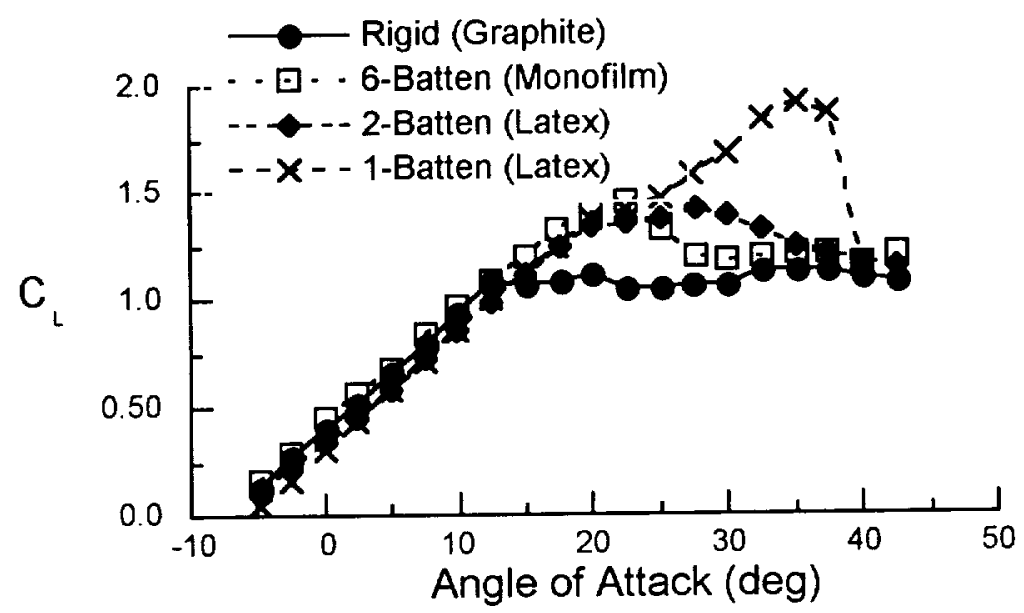

Figure 9. Lift coefficient vs. angle of attack for configurations with varying wing stiffness (power off). 


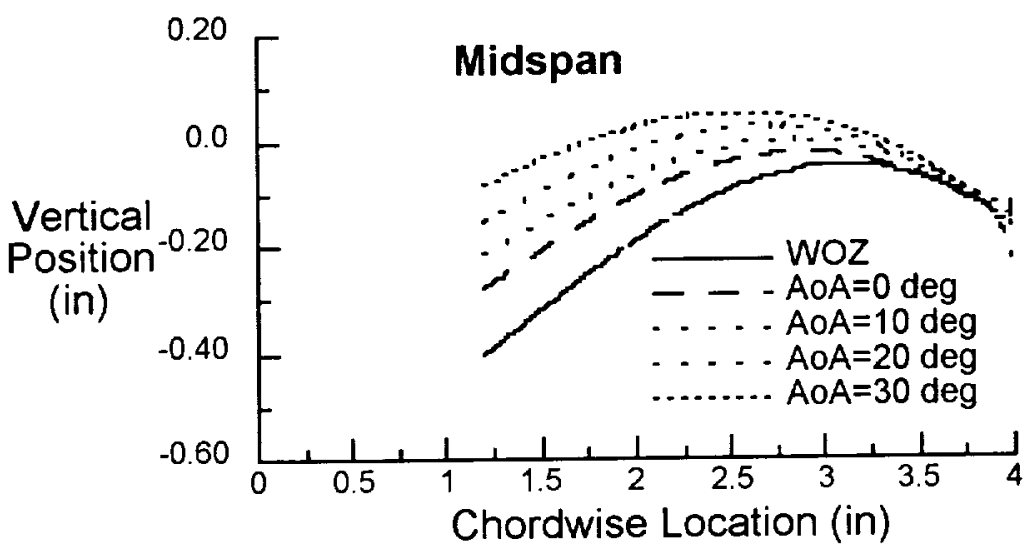

Figure 10. Wing camber of two-battened latex configuration for range of angles of attack ( $q=1.6 \mathrm{psf}$, trim power).

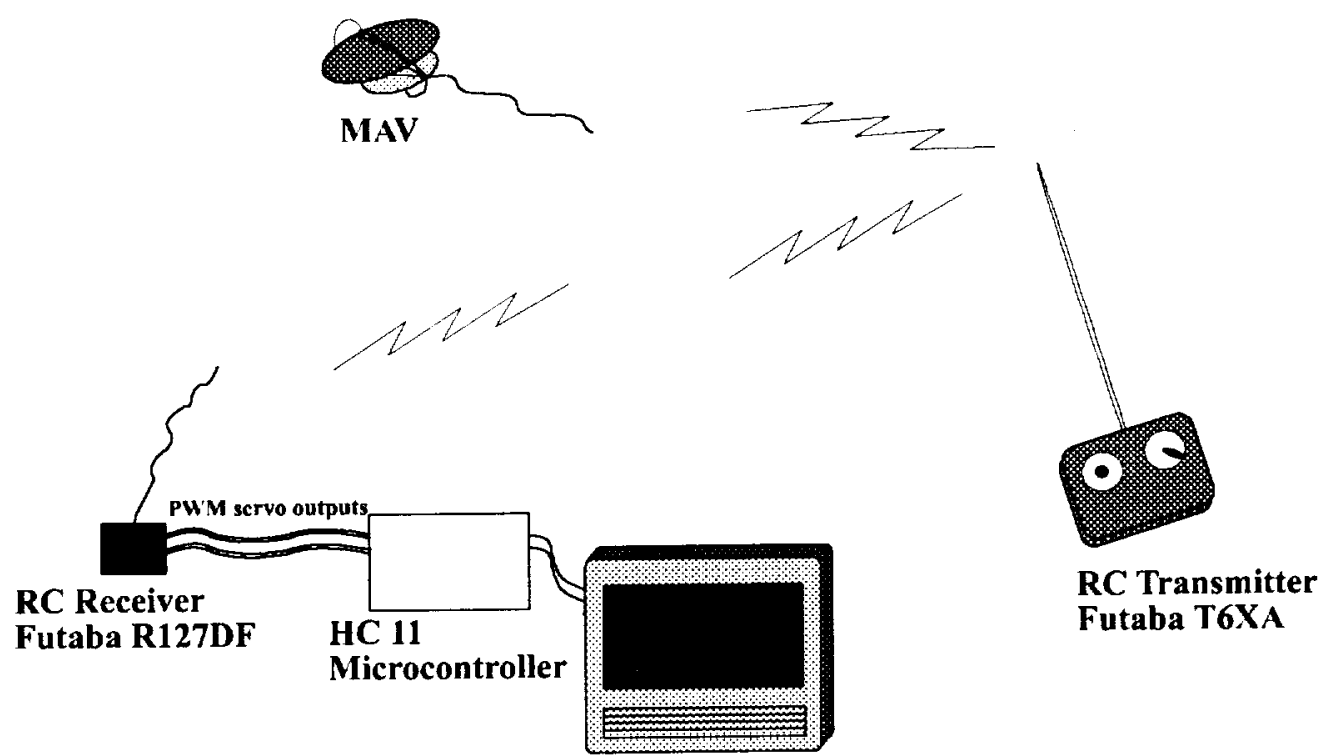

Notebook PC

Figure 11. Flight control input data recording system. 
Case 1: Flexible Wing Forward CG

Case 2: Flexible Wing Aft CG

Case 3: Rigid Wing Forward CG

Case 4: Flexible Wing Forward CG - Gusty
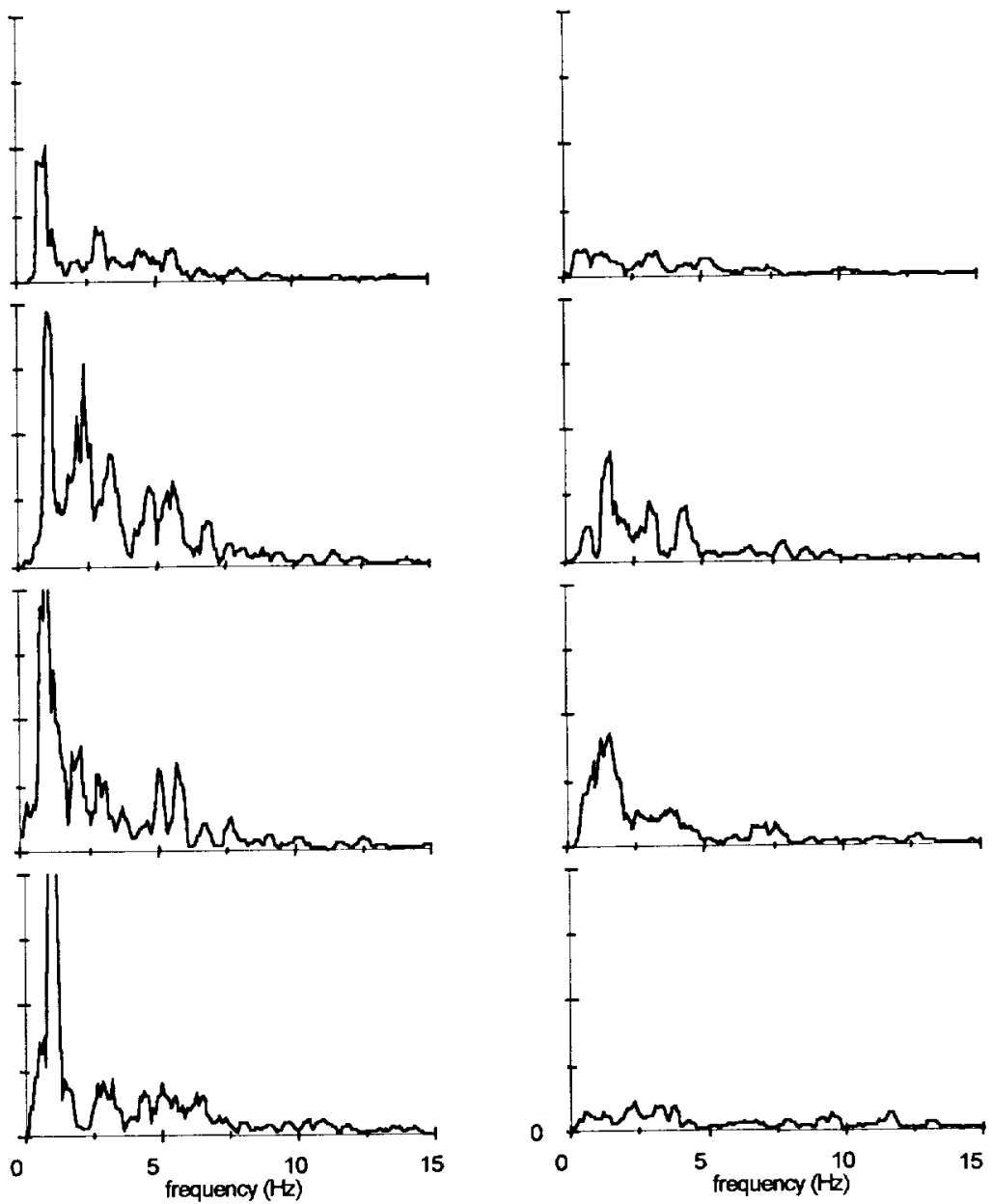

Figure 12. Autospectra of elevator movement rate data and roll input rate data. The vertical axis is the relative power at each frequency. The curves are generated by processing the differentiated elevator and roll input data ( 512 data points ach case). 